\title{
Descripción de gemelos siameses de Bubalus bubalis bubalis (Búfalo), Sucre, Colombia
}

\section{Description of Siamese twins of Bubalus bubalis bubalis (Buf- falo), Sucre, Colombia}

\author{
Jaime De La Ossa V, ${ }^{1 *}$ Ph. D, Luis Altahona $B,{ }^{1}$ Zootec. \\ ${ }^{1}$ Universidad de Sucre, Facultad de Ciencias Agropecuarias. Grupo de Investigación en \\ Biodiversidad Tropical. Sincelejo, Sucre, Colombia. Correspondencia: \\ jaimedelaossa@yahoo.com
}

Recibido: Octubre 19 de 2009; Aceptado: Abril 28 de 2010.

\begin{abstract}
RESUMEN
El presente trabajo muestra el hallazgo de gemelos siameses de Bubalus bubalis bubalis (Murrah x Nili Ravi) en una la finca, dedicada a la explotación de esta especie de ganado y ubicada en el municipio de San Marcos, departamento de Sucre, Colombia. Los neonatos siameses se identificaron por las características externas con respecto al tipo de unión como isquiópagos sincéfalos. Se presentan datos de la necropsia y se describen órganos y estructuras internas de los animales.
\end{abstract}

Palabras clave: Bubalus bubalis bubalis, gemelos siameses, isquiópagos, Colombia.

\begin{abstract}
The present paper reports of Siamese twins Bubalus bubalis bubalis (Murrah $\mathrm{x}$ Nili Ravi) on a farm, dedicated to the exploitation of this species of livestock and located in the municipality of San Marcos, Sucre Department, Colombia. The Siamese infants were identified by external characteristics regarding the type of union as isquiópagos sincéfalos. Data of the autopsy and a description of organs and internal structures of animals are showed.
\end{abstract}

Key words: Bubalus bubalis bubalis, siamese twins, ischiiopagous, Colombia. 


\section{NTRODUCCIÓN}

El origen de los gemelos siameses aún no está bien definido, se indica que son el resultado de una aberración en el proceso de formación de los gemelos monocigóticos; ellos son en sí mismos considerados una particularidad del desarrollo embrionario normal (1). Se considera que este tipo de malformaciones son conocidas desde la antigüedad y se les registra por su carácter evidente y el asombro espectacular que causan. No obstante, cuando se les adjudica el calificativo de siameses, representa un defecto severo en el cual uno de los gemelos uniovular falló en su exitosa separación; la separación incompleta se considera como el resultado de una duplicación parcial que ocurre en el disco embrionario antes de la tercera semana de vida intrauterina (2).

El grado y origen de la duplicación determina el tipo de gemelos siameses, puede variar desde los que sólo están conectados por una fina membrana, hasta dos o más individuos más o menos formados completamente con un solo tronco y duplicación, bien de la cabeza o sólo de la parte caudal del cuerpo. No existen factores conocidos que predispongan esta malformación, aunque se señala que puede deberse a: defectos propios de la línea primitiva, o envejecimiento del óvulo que puede resultar por disminución de la capacidad de diferenciación normal, formando dos centros de diferenciación, ninguno capaz de suprimir al otro $(2,3)$. También está en discusión, como variables incidentes, los factores genéticos, dificultades nutricionales de la madre y problemas de desarrollo del embrión debido a infecciones o deficiente irrigación sanguínea $(3,4)$. Ademas es importante anotar, que los siameses tienen elevada incidencia de otras patologías, muchas pueden estar relacionadas con el sitio de unión, pero otras no están anatómicamente conectadas (3).

Embriológicamente existe de forma normal una completa separación de las primeras blastómeras seguida de una fusión parcial lo que produciría gemelos normales, se asume que en los siameses se da una fisión, que se manifiesta en una incompleta separación de las primeras dos blastómeras, con duplicación exclusiva de aquellas partes que se separaron completamente, lo que permite concluir que son el resultado de una incompleta separación del disco embrionario cerca del final de la segunda semana del desarrollo (3).

Desde el punto de vista de su clasificación, los siameses dobles están constituidos por dos individuos coalescentes o fusionados en diverso grado y en diferentes regiones corporales. Pueden ser simétricos o asimétricos, en esta última condición uno de los individuos está bien conformado y se le denomina autocito, mientras el otro es rudimentario y recibe la denominación de parásito (5). Pueden ser teratópagos que son aquellos que están unidos en una extensión más o menos grande: cefalópagos (cabeza), isquiópagos (pelvis), pigópagos (región lumbosacra), onfalópagos (ombligo), xifópagos (apéndice xifoides de esternón), toracópagos (comunicados por cavidades torácicas) (4).

\section{PRESENTACI ÓN DEL CASO}

Lugar de registro. El nacimiento tuvo lugar en la Hacienda Mosquito, Municipio de San Marcos, departamento de Sucre, Colombia (834'55' N, 7508'06' O). Dicha zona ambientalmente se clasifica como bosque seco tropical (6). No obstante, por sus características climatológicas se le puede destacar sectorialmente como una amplia zona de humedales, perteneciente a la región de la Mojana, específicamente situada en la zona del bajo río San J orge.

Descripción parental. Padre, edad 10 años, raza Murrah, proveniente de Plato Magdalena, Colombia. Peso 680 kg, tiempo de incorporación al hato 2 años, y se mantiene en una relación macho: hembras de 1:80. Madre, edad 5,8 años, raza NiliRavi, proveniente de Montelibano, Córdoba, Colombia. Peso 520 kg., tiempo de incorporación al hato 4 años, multípara con tres partos normales anteriores.

Descripción del neonato. Parto distócico, de fecha 25 de junio de 2009, peso total de 
los gemelos siameses $60 \mathrm{~kg}$. Macroscópicamente se observó que los gemelos malformados estaban unidos en la porción media del abdomen fusionados desde la región pélvica hasta el ombligo (Figura 1A), poseían una sola pelvis (Figura 1B), un cordón umbilical, un pene y dos sacos escrotales; con dos cabezas perfectamente formadas, dos cajas torácicas, cuatro extremidades anteriores y dos extremidades posteriores, dos anos ciegos y dos colas (Figura 1C). Durante la necropsia se evidenció que
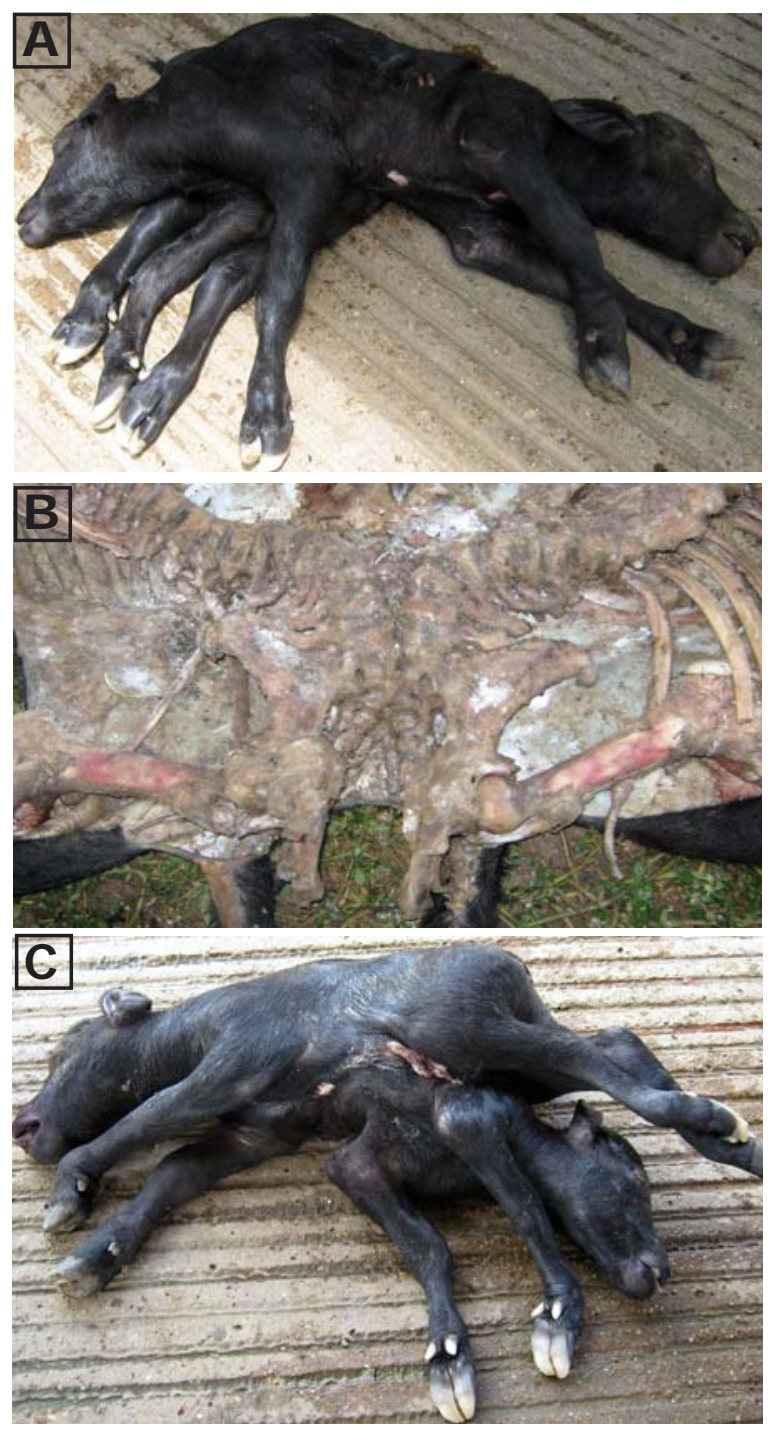

Figura 1. Vista lateral de los gemelos siameses de Bubalus bubalis $(A)$, fusión pélvica que tipifica los gemelos siameses isquiópagos registrados en este estudio (B), vista latero-ventral de los gemelos isquiópagos de Bubalus bubalis indicando lugar de unión (C). compartían el hígado y el sistema urogenital, existiendo duplicidad de los demás órganos internos.

\section{DISCUSI ÓN}

El tipo de unión existente a nivel de huesos pélvicos comunes, permite identificar la malformación congénita como isquiópaga, al poseer dos cabezas perfectamente formadas se les puede identificar secuencialmente como sincéfalos $(4,5)$.

La hacienda Mosquito, lugar de registro de esta malformación, posee un hato de 1.032 búfalos, con un manejo desde hace 5 años aproximadamente, es la primera vez que se presenta este tipo de nacimiento, lo que hace que en relación con las hembras aptas para reproducción se tenga una ocurrencia del $0.31 \%$, valor que está por debajo del $2 \%$ de ocurrencia establecida (6).

Los gemelos siameses son considerados una patología rara que afecta a 1:200 embarazos de gemelos monocigóticos, 1: 900 embarazos gemelares y $1: 25.000$ a 100.000 nacimientos (7). Son más observados en rumiantes que en otros grupos zoológicos. El registro aquí presentado es del tipo de malformación de siameses de menor ocurrencia, ya que los toracópagos representan el $49 \%$, los onfalópagos $35 \%$, los pyópagos $18 \%$, los cefalópagos $2 \%$ junto a los isquiópagus con otro $2 \%(5)$.

Se demuestra que este tipo de patología posee suficientes registros, se sabe que puede presentarse en diferentes grupos zoológicos, por ejemplo en mamíferos (710); específicamente para búfalo murrah se tiene hallazgo de dicephalus dipus dibrachious (6), y para búfalos en general existen registros de este tipo de malformación en diferentes publicaciones (11); entre los anfibios y reptiles también se presentan los gemelos siameses (12).

Se indica que no existen factores conocidos que predispongan la ocurrencia de este tipo de malformaciones congénitas; 
pero pueden ser asociadas con envejecimiento ovular (13), edad de los animales (14). Esta situación no podria darse en este caso, ya que se trataba de una hembra de 5,8 años, con tres partos anteriores y en perfecto estado reproductivo y óptima condición corporal. Se le podria relacionar con problemas nutricionales y deficiente irrigación sanguínea, factores que no son evidentes en este hallazgo (14).
Por ultimo quedarían las variables genéticas $(9,10)$, como la endogamia posible de consanguinidad existente entre los padres, bien sea por la limitada diferencia geográfica de su procedencia, por similitud de los individuos importados inicialmente o grupo fundador y por la ausencia de nuevos reproductores lejanamente emparentados. Lo anterior podria explicar la pérdida de variabilidad genética por consanguinidad y por lo tanto la presencia de ciertas malformaciones o anomalías genéticas.

\section{REFERENCIAS}

1 Nottidge HO, Omobowale TO, Olopade JO, Oladiran OO, Ajala OO. A Case of Craniothoracopagus (Monocephalus Thoracopagus Tetrabrachius) in a Dog. Anat Histol Embryol 2007; (36): 179-181.

2. Lattus J, Almuna R, Paredes A. Siameses o gemelos unidostoracoonfalópagos y revisión bibliográfica nacional e internacional. Rev Chil Obstet Ginecol 2002; 67(5):392-401.

3 Ingar J, Huertas E, Mezarina F, Gutiérrez $\mathrm{G}$, Ordemar P. $\mathrm{S}$ i a $\mathrm{m}$ e $\mathrm{s}$ e $\mathrm{s}$ : presentación de un caso. Rev Per Ginecol Obstet 2007; 53:213-216.

4.. López-Gómez JR, Rivas GM, Alvarado S, Castro J. Gemelos unidos: presentación de un caso de malformación fetal: Teratódimo, opódimo, tetraoftalmos, rinodimo. Rev Obstet Ginecol Venezol 2000; 60(1):59-61.

5. Dickinson JE. Monoamniotic twin pregnancy: a review of contemporary practice. Aust N Z J Obstet Gynaecol 2005; 45:474-478.

6. Otero-Alvares E, Mosquera-Aguirre L. SilvaCastro G, Guzmán-Victoria J, editores. Bosque seco tropical Colombia. Cali, Colombia: Banco de occidente; 2006.
7. Srivastava A, Kumar A, Maurya SK, Singh A, Singh VK. Adicephalus monster in murrah buffalo. Buffalo Bulletin 2008; 27(3): 231-233.

8 Mostafa MB, Koma KLM, Acon J. Conjoined twin piglets. Vet Rec 2005; 156: 779-780.

9 Corbera JA, Arencibia A, Morales I, Gutierrez C. Congenital duplication of the caudal region (monocephalus dipygus) in a kid goat. Anat Histol Embryol 2005; (34):61-63.

10. Oviedo ST, González TM, Oviedo PM. Monocephalus dipygus en achorros: reporte de dos casos. Rev MVZ Córdoba 2005; 13(3): 1522-1525.

11. Lotfy M, Ibrahim IM, Sakr S, Ayoub B, Tha A, Nawito M, Mohamoud KGh. Succesful separation on Craniopagus parasiticus in an egyptian buffalo calf. Global Vet 2009; 3(3): 193-198.

12 Fronda RC, Andres IO. Two Souls, One Heart, One Body: A Case Report. Philippine J Sci 2009; 42(1):25-31. 
13. Platt MJ, Marshall A, Pharoah PO. The effects of assisted reproduction on the trends and zygosity of multiple in England and Wales 1974- 99. Twin Res 2001; $4(6): 417-421$.
14. Mahomoud KGh, Mobarak MS, Farghaly AA. Effect of dietary restricction on genetic material and reproductive performance in rabbit. Egypt J Genet Cytologia 2006; 35: 129-143. 